\title{
Diagnóstico do Oligoâmnio pela Ultra-Sonografia: Uso de Diferentes Medidas do Maior Bolsão Comparadas ao ILA
}

\author{
Diagnostic Capacity of Oligohydramnios by Ultrasound Using Different Measures \\ of the Maximum Pool Depth of Amniotic Fluid in Comparison to AFI
}

Maria Regina Machado Perrotti, José Guilherme Cecatti, Milton Bricola Filho Kleber Cursino de Andrade, Fernanda Fioravanti Azank dos Santos

\section{RESUMO}

Objetivo: comparar a capacidade de diagnosticar oligoâmnio pela ultra-sonografia por meio de diferentes valores do maior bolsão de líquido amniótico, em comparação ao índice de líquido amniótico (ILA), em gestantes normais, de 36 a 42 semanas de gestação.

Métodos: realizou-se um estudo descritivo de validação de método diagnóstico, incluindo 875 gestantes normais. Mediante um exame ultra-sonográfico obstétrico de rotina, foi feita a medida do maior bolsão de líquido amniótico para o diagnóstico de oligoâmnio, utilizando como padrão-ouro o índice de líquido amniótico. Os dados foram analisados por meio do cálculo da sensibilidade e da especificidade da medida do maior bolsão de líquido amniótico, utilizando os diferentes pontos de corte de 10, 20 e $30 \mathrm{~mm}$, em comparação aos valores normais do indice de líquido amniótico determinados pelos percentis 2,5 e 10 nas respectivas idades gestacionais.

Resultados: a medida do maior bolsão de líquido amniótico apresenta baixa sensibilidade para diagnosticar oligoâmnio quando se adotam os pontos de corte 10 e $20 \mathrm{~mm}$, e boa sensibilidade e especificidade quando se adota $30 \mathrm{~mm}$, quando comparadas às medidas do indice de liquido amniótico nos percentis 2,5 e 10 da curva normal. A sensibilidade e especificidade da medida do maior bolsão são melhores, quando se adota o ponto de corte de $30 \mathrm{~mm}$ para diagnosticar oligoâmnio em comparação ao percentil 2,5.

Conclusões: a capacidade de diagnosticar oligoâmnio pela medida do maior bolsão é satisfatória apenas com o ponto de corte em $30 \mathrm{~mm}$.

PALAVRAS-CHAVE: Oligoâmnio. Índice de líquido amniótico (ILA). Gravidez normal.

\section{Introdução}

Há décadas que se tenta avaliar, por meio de exames de monitorização biofísica fetal, até que ponto o feto estaria mais seguro no ambiente intra-útero que externamente, sob a assistência neonatal. É provável que esta dúvida permaneça sem resposta em muitas situações, mas o aperDepartamento de Tocoginecologia - Faculdade de Ciências Médicas

Universidade Estadual de Campinas

Correspondência:

José Guilherme Cecatti

Caixa Postal 6030

13.081.970 - Campinas - SP

Fax: (19) 788-9304

Este estudo foi financiado pela FAPESP (processo 97/05382-4) feiçoamento de novas técnicas de investigação do bem-estar fetal vem trazendo melhores resultados perinatais, além de auxiliar na compreensão da complexa interação fisiológica e patológica entre os organismos materno e fetal. Um marco fundamental na avaliação fetal deve-se à utilização do ultra-som, com origem em pesquisas realizadas durante a I Guerra Mundial, atualmente incorporado à rotina propedêutica em Obstetrícia ${ }^{1}$.

Especificamente em relação ao volume de líquido amniótico, já está bem estabelecida sua relação com o bem-estar fetal, pois sua variação pode estar associada a processos patológicos fetais e/ou maternos ${ }^{2,3}$. Conseqüentemente, a diminuição do volume de líquido amniótico está intimamente correlacionada com um aumento das ta- 
xas de morbidade e mortalidade perinatal ${ }^{2,3}$.

A quantificação do volume de líquido amniótico tem sido, desde os primeiros estudos na década de 60 , objeto de investigação, no intuito de se determinarem valores abaixo dos quais se poderia denominar oligoâmnio, e acima, polidrâmnio. No passado, a estimativa do volume de líquido amniótico era realizada pela palpação abdominal e medida da altura uterina. Os primeiros investigadores que quantificaram o volume de líquido amniótico no momento da histerotomia e em abortos observaram um aumento progressivo no periodo entre a $10^{\mathrm{a}}$ e $20^{\mathrm{a}}$ semana de gestação ${ }^{4}$. Posteriormente, Gadd ${ }^{5}$ correlacionou estes dados com os de técnicas de diluição de corantes, mostrando um aumento de líquido amniótico até a 30a semana, permanecendo estável de 30 a 37 semanas e, após, apresentando um declínio gradual. Após a $42^{\text {a }}$ semana, demonstrou-se haver um rápido declínio em seu volume ${ }^{6}$.

A variação de volume do líquido amniótico começou a ser melhor avaliada após o advento da ultra-sonografia, quando técnicas qualitativas e semiquantitativas foram desenvolvidas ${ }^{7}$. Dentre os métodos qualitativos, o mais importante é a análise subjetiva do observador quanto aos múltiplos lagos de líquido amniótico. No entanto, esta análise depende da experiência do observador e está sujeita a grande variabilidade, tanto inter quanto intra-observador.

Os métodos semiquantitativos determinam o volume de líquido amniótico pela medida da profundidade ou largura do maior bolsão do mesmo ${ }^{8}$, ou pela técnica dos quatro quadrantes descrita por Phelan et al. ${ }^{9}$, denominada índice de líquido amniótico (ILA). Este índice é obtido pela soma das profundidades do maior bolsão de cada um dos quadrantes do útero, determinados pela divisão imaginária do útero pela linea nigra longitudinalmente e perpendicularmente por uma linha que passa pela cicatriz umbilical. Ambos os métodos mostraram boa correlação entre as medidas e o volume verdadeiro de líquido amniótico, quando comparados à medição direta do mesmo, pela técnica da diluição de solução de para-aminohipurato a $10 \%$ por amniocentese ${ }^{10}$.

Pode-se utilizar ainda a medida do maior bolsão de líquido amniótico, considerando-se, pela definição de Manning et al. ${ }^{8}$, oligoâmnio quando a medida do maior e único bolsão for menor que 1 $\mathrm{cm}$; quando medir de 8 a $12 \mathrm{~cm}$, polidrâmnio discreto; de 12 a $16 \mathrm{~cm}$, polidrâmnio moderado, e acima de $16 \mathrm{~cm}$, polidrâmnio grave. A acurácia deste método parece ser equivalente à avaliação subjetiva do líquido amniótico ${ }^{11}$. Moore ${ }^{12}$ comparou a medida do maior bolsão com o ILA, encontrando uma menor capacidade de detectar casos de oligoâmnio pela primeira técnica (sensibilidade de
$42 \%$ e valor preditivo positivo de $51 \%$ ).

$\mathrm{Na}$ década passada, descreveu-se uma técnica ultra-sonográfica para avaliação da vitalidade fetal, conhecida como perfil biofisico fetal (PBF). Aplicando-a na prática, seu idealizador diagnosticou alterações da vitalidade fetal com alta especificidade, com uma taxa de falso-negativos extremamente baixa, de 0,6 a 0,7/1.000, associadas a um índice de mortalidade perinatal de $1 / 1.000^{13}$. O PBF consiste na análise de cinco parâmetros, sendo quatro biológicos fetais (movimentos corpóreos e respiratórios, reatividade cardíaca fetal, tônus) que refletem a atividade do sistema nervoso central e, indiretamente, seu estado de oxigenação ${ }^{14}$ e a quantidade de líquido amniótico em um bolsão para avaliar se o processo é crôni$\mathrm{co}^{15}$. Ao apresentar movimentos corpóreos e respiratórios, preservação do tônus e reatividade cardiaca aos movimentos, o feto recebe dois pontos para cada item. Da mesma forma, se apresentar líquido amniótico em um bolsão com medida maior que $20 \mathrm{~mm}$ de diâmetro, também recebe dois pontos. Caso esses critérios não sejam cumpridos, não recebe pontos (escore zero).

Alguns autores indicam interrupção da gestação baseados na diminuição do volume do líquido amniótico no PBF, mesmo que os outros parâmetros estejam normais ${ }^{8}$. Os padrões de normalidade do líquido amniótico, no PBF, são avaliados pela presença de pelo menos uma bolsa de líquido amniótico com mais de $2 \mathrm{~cm}$ de diâmetro, medida em dois planos perpendiculares ${ }^{14}$. Permanece, contudo, a dúvida se este valor de maior bolsão de líquido amniótico é mais apropriado para se diagnosticar oligoâmnio pelas evidentes repercussões em termos de decisões clínicas e resultados perinatais.

\section{Pacientes e Métodos}

O presente estudo é descritivo, de validação de método diagnóstico. Foram avaliadas no serviço de Ecografia do Centro de Atenção Integral à Saúde da Mulher, da Universidade Estadual de Campinas, e no serviço médico "Medical Center - Diagnose Serviço de Ecografia e Monitorização Fetal", ambos de Campinas, Estado de São Paulo, 875 gestantes normais, da 36 à $42^{\mathrm{a}}$ semana de gestação, no período de agosto de 1997 até abril de 1998. A avaliação foi feita por um exame ultra-sonográfico obstétrico de rotina, com a medida do maior bolsão de líquido amniótico, utilizando como padrão-ouro o cálculo do índice de líquido amniótico, pela técnica de Phelan et al. ${ }^{9}$, modificada por Jeng et al. ${ }^{16}$.

Apenas os casos com data da última menstruação conhecida com certeza ou, caso esta pri- 
meira condição não fosse cumprida, com idade gestacional estimada por exame ultra-sonográfico pélvico precoce, realizado antes da $24^{a}$ semana, foram incluídos; foram consideradas elegiveis apenas as mulheres sem uso de anticoncepcionais orais nos últimos três meses ou injetáveis nos últimos seis meses anteriores a esta gestação, para que a idade gestacional fosse considerada confiável.

Os casos considerados como gestação nãonormal, com hipertensão arterial crônica, hipertensão gestacional, diabetes mellitus, macrossomia fetal, rotura de membranas, senescência placentária precoce (segundo classificação de Grannum et al. ${ }^{17}$ ), gestação múltipla, retardo de crescimento intra-uterino, anomalia congênita fetal, óbito fetal ou isoimunização fetal foram, excluídos. Outras situações tais como doenças metabólicas, nefropatias, cardiopatias, hiper e hipotireoidismo materno também foram excluídas.

Para a realização dos exames ultrasonográficos, utilizaram-se os aparelhos Toshiba SSA- $140^{\circledast}$ e Acuson XP4A ${ }^{\circledast}$, com um transdutor convexo de 3,75 mHz. Todos os exames foram realizados somente por um examinador.

Os maiores bolsões de cada quadrante na profundidade foram medidos estando a sonda perpendicular ao plano e alinhada longitudinalmente, com a gestante em posição supina. O ILA foi obtido pela soma das medidas, em milimetros, dos bolsões dos quatro quadrantes. Na medida do maior bolsão de líquido amniótico não foram incluídas partes fetais e alças de cordão umbilical, estas últimas bem visiveis ao mapeamento colorido, usado em caso de dúvida.

Os dados foram analisados por meio do cálculo da sensibilidade e da especificidade da medida do maior bolsão de líquido amniótico, utilizando os diferentes pontos de corte de 10,20 e $30 \mathrm{~mm}$, em comparação aos valores normais do índice de líquido amniótico determinados pelos percentis 2,5 e 10 nas respectivas idades gestacionais (Tabela 1), segundo a curva de Perrotti ${ }^{18}$. O estudo foi aprovado pelo Comitê de Ética em Pesquisa da instituição.

Tabela 1 - Valores estimados ajustados para os percentis 2,5 e 10 do índice de líquido amniótico, segundo a idade gestacional.

\begin{tabular}{ccc}
\hline $\begin{array}{c}\text { Idade Gestacional } \\
\text { (sem) }\end{array}$ & $\begin{array}{c}\text { Percentil 2,5 } \\
(\mathbf{m m})\end{array}$ & $\begin{array}{c}\text { Percentil 10 } \\
(\mathbf{m m})\end{array}$ \\
\hline 36 & 62,1 & 90,7 \\
37 & 55,6 & 84,3 \\
38 & 48,7 & 77,4 \\
39 & 41,3 & 69,8 \\
40 & 33,5 & 61,6 \\
41 & 25,3 & 52,9 \\
42 & 16,6 & 43,5 \\
\hline
\end{tabular}

Modificado de Perrotti ${ }^{18}$.

$\mathrm{RBG0}-$ v. $22, n^{0} 1,2000$

\section{Resultados}

Adotou-se, primeiramente, como padrãoouro o índice de líquido amniótico, e como valores de normalidade, aqueles acima do percentil 2,5 da curva de ILA. Compararam-se então as medidas do maior bolsão, categorizadas pelos pontos de corte 10,20 e $30 \mathrm{~mm}$, para diagnosticar oligoâmnio. O diagnóstico de oligoâmnio comparativamente entre os dois métodos está na Tabela 2. Nesta situação, a sensibilidade deste segundo método variou de 7,10 a 35,90\% em média, para os pontos de 10 e $20 \mathrm{~mm}$. Quando se adotaram os valores acima do ponto de corte de $30 \mathrm{~mm}$ como representativos de normalidade, a sensibilidade da medida do maior bolsão aumentou de maneira importante, de 75 a 100\%, em função da idade gestacional (Tabela 3).

Com relação à curva de ILA utilizando o percentil 10, o diagnóstico de oligoâmnio comparativamente entre os dois métodos também está na Tabela 2. As sensibilidades do maior bolsão aumentaram com o aumento do valor do ponto de corte, mas foram sempre inferiores àquelas comparadas ao percentil 2,5 (Tabela 4).

Quanto à especificidade, ela foi praticamente de $100 \%$ em todas as idades gestacionais estudadas, tanto para os valores dos percentis 2,5 quanto 10 , quando se adotaram como pontos de corte 10 e $20 \mathrm{~mm}$. A especificidade apresentou apenas um pequeno decréscimo com o ponto de corte de $30 \mathrm{~mm}$ para o maior bolsão.

Tabela 2 - Distribuição das mulheres com diagnóstico de oligoâmnio pelo ILA segundo percentis e diagnóstico pela medida do maior bolsão com diferentes pontos de corte.

\begin{tabular}{cccrrr}
\hline ILA & $\begin{array}{c}\text { Oligoâmnio pelo } \\
\text { maior bolsão } \\
\text { Percentis }\end{array}$ & $\begin{array}{c}\text { Oligoâmnio pelo ILA } \\
\text { Pontos de corte } \\
(\mathbf{m m})\end{array}$ & Sim & Não & \\
\hline Percentil 2,5 & 10 & Sim & 2 & - & 2 \\
& 20 & Não & 26 & 847 & 873 \\
Percentil 10 & \multirow{2}{*}{30} & Não & 25 & 834 & 859 \\
& 10 & Sim & 21 & 37 & 58 \\
& & Não & 7 & 810 & 817 \\
& 20 & Sim & 2 & - & 2 \\
& & Sim & 73 & 800 & 873 \\
& 30 & Não & 59 & - & 16 \\
& & Sim & 400 & 859 \\
Não & 35 & 782 & 817 \\
\hline
\end{tabular}


Tabela 3 - Sensibilidade (S) e especificidade (E) de diferentes valores do maior bolsão de ILA para o diagnóstico de oligoâmnio pelo ILA, segundo idade gestacional (percentil 2,5 da curva de ILA por idade gestacional).

\begin{tabular}{ccccccc}
\hline $\begin{array}{c}\text { Maior bolsão }(\mathbf{m m}) \\
\text { Idade gestacional }(\mathbf{s e m})\end{array}$ & $\mathbf{S}$ & $\mathbf{1 0}$ & $\mathbf{S}$ & $\mathbf{E}$ & $\mathbf{3}$ & $\mathbf{3 0}$ \\
\hline 36 & - & 100,0 & - & 100,0 & - & 100,0 \\
37 & - & 100,0 & 20,0 & 100,0 & 100,0 & 97,6 \\
38 & - & 100,0 & - & 100,0 & - & 98,3 \\
39 & - & 100,0 & - & 100,0 & 75,0 & 97,4 \\
40 & 16,0 & 100,0 & 33,3 & 100,0 & 83,3 & 94,8 \\
41 & 14,3 & 100,0 & 57,1 & 98,2 & 100,0 & 93,8 \\
42 & - & 100,0 & 33,3 & 100,0 & 100,0 & 86,5 \\
Total & $7,1 \%$ & $100,0 \%$ & $35,9 \%$ & $99,8 \%$ & $75,0 \%$ & $95,6 \%$ \\
\hline
\end{tabular}

Tabela 4 - Sensibilidade (S) e especificidade (E) de diferentes valores do maior bolsão de ILA para o diagnóstico de oligoâmnio pelo ILA, segundo idade gestacional (percentil 10 da curva de ILA por idade gestacional).

\begin{tabular}{ccccccc}
\hline $\begin{array}{c}\text { Maior bolsão }(\mathbf{m m}) \\
\text { Idade gestacional }(\mathbf{s e m})\end{array}$ & $\mathbf{S}$ & $\mathbf{E}$ & $\mathbf{S}$ & $\mathbf{E}$ & $\mathbf{3}$ & $\mathbf{3 0}$ \\
\hline 36 & - & 100,0 & - & 100,0 & - & 100,0 \\
37 & - & 100,0 & 16,7 & 100,0 & 83,3 & 97,6 \\
38 & - & 100,0 & - & 100,0 & 22,2 & 100,0 \\
39 & - & 100,0 & - & 100,0 & 55,5 & 99,1 \\
40 & 11,1 & 100,0 & 55,5 & 100,0 & 66,7 & 95,5 \\
41 & 5,8 & 100,0 & 35,3 & 100,0 & 64,7 & 97,1 \\
42 & - & 100,0 & 33,3 & 100,0 & 91,7 & 94,4 \\
Total & $2,7 \%$ & $100,0 \%$ & $21,3 \%$ & $100,0 \%$ & $53,3 \%$ & $97,7 \%$ \\
\hline
\end{tabular}

Os valores preditivos positivo e negativo dos pontos de corte 10,20 e $30 \mathrm{~mm}$ estão expressos na Tabela 5, nos respectivos valores do percentil 2,5 e 10 da curva de ILA.

Tabela 5 - Valores preditivos positivo (VPP) e negativo (VPN) de diferentes valores do maior bolsão de ILA para o diagnóstico de oligoâmnio pelo ILA, segundo idade gestacional (percentil 2,5 e 10 da curva de ILA por idade gestacional).

\begin{tabular}{clllll}
\hline & \multicolumn{2}{c}{ Percentil 2,5 } & & \multicolumn{2}{c}{ Percentil 10 } \\
\cline { 2 - 3 } \cline { 6 - 7 } Pontos de corte (mm) & VPP & VPN & & VPP & VPN \\
\hline 10 & 1,0 & 0,97 & & 1,0 & 0,92 \\
20 & 0,87 & 0,97 & & 1,0 & 0,93 \\
30 & 0,43 & 0,99 & & 0,69 & 0,96 \\
\hline
\end{tabular}

\section{Discussão}

A melhor maneira de se quantificar o oligoâmnio e os valores limitrofes a se adotar ainda continua incerta. Visando o bem-estar fetal e a preservação da vitalidade fetal, os métodos diag- nósticos ecográficos para avaliação do volume de líquido amniótico, entre eles o índice de líquido amniótico e a medida do maior bolsão, utilizamse de variáveis diferentes para tentar predizer o risco fetal pela diminuição do volume de líquido amniótico.

Tentou-se avaliar a correspondência entre estes dois métodos para diagnosticar oligoâmnio, com a determinação do ponto de corte com melhor sensibilidade e especificidade. Para o obstetra, isto significa como interpretar um perfil biofisico em que o feto recebeu pontuação "dois" para a variável "líquido amniótico" pela medida do maior bolsão, mas apresenta um diagnóstico de oligoâmnio pelo ILA.

Como já demonstrado anteriormente pelos estudos de Moore $^{12}$, a capacidade de detectar oligoâmnio pela medida do maior bolsão parece ser menor em relação à medida do ILA. No entanto, como há divergências quanto ao ponto de corte do valor de maior bolsão a se adotar, compararam-se os já preconizados e utilizados na prática até o momento, de $10 \mathrm{~mm}^{15}$, de $20 \mathrm{~mm}$ (modificação proposta por Manning, et al. ${ }^{13}$ ) e de $30 \mathrm{~mm}^{19}$. 
Os presentes resultados são concordantes com a literatura em mostrar que a sensibilidade e a especificidade para diagnosticar oligoâmnio são menores quando se adotam como pontos de corte 10 e $20 \mathrm{~mm}$ de medida do maior bolsão. Assim, o valor limitrofe de $30 \mathrm{~mm}$ de um único bolsão de líquido amniótico no perfil biofísico fetal teria melhor capacidade para diagnosticar oligoâmnio que os limites de 10 e $20 \mathrm{~mm}$, quando comparados à medida do ILA.

Desta forma, a medida do maior bolsão pode, em algumas circunstâncias, não refletir globalmente o volume de líquido amniótico, que pode estar diminuído pela medida do ILA quando a medida do maior bolsão encontra-se normal (falsonegativo para a medida do maior bolsão). Talvez fosse interessante que se quantificassem as duas medidas (maior bolsão e ILA), sempre que fosse solicitado perfil biofísico fetal. O obstetra teria então uma visão mais global da situação da vitalidade fetal, principalmente no que concerne ao volume de líquido amniótico, com informações mais precisas para a tomada de decisões clínicas.

Concluindo, a medida do maior bolsão de líquido amniótico apresenta baixa sensibilidade para diagnosticar oligoâmnio quando se adotam como pontos de corte 10 e $20 \mathrm{~mm}$, e boa sensibilidade e especificidade quando se adota $30 \mathrm{~mm}$, comparando-se às medidas do índice de liquido amniótico nos percentis 2,5 e 10 da curva de Perrotti ${ }^{18}$. A sensibilidade e especificidade da medida do maior bolsão são melhores, contudo, adotando-se o ponto de corte de $30 \mathrm{~mm}$ para diagnosticar oligoâmnio quando se adotam valores do percentil 2,5 .

Assim, a capacidade em diagnosticar oligoâmnio pela medida do maior bolsão é satisfatória apenas com o ponto de corte de $30 \mathrm{~mm}$ e este deveria ser o utilizado em avaliações sistemáticas do perfil biofisico fetal.

\section{SUMMARY}

Purpose: to compare the capacity of diagnosing oligohydramnios by ultrasound using different measures of the maximum pool depth of amniotic fluid in comparison to the amniotic fluid index among normal pregnant women from the 36th to the 42nd week of gestation.

Methods: a descriptive study of diagnostic validity was perfomed, on 875 normal pregnant women who were studied through routine obstetric ultrasound examinations, including the measure of the maximum pool depth for the diagnosis of oligohydramnios, using the amniotic fluid index as the gold standard. The data were analyzed through sensitivity and specificity of the maximum pool depth of amniotic fluid using 10,20 and $30 \mathrm{~mm}$ cut-offs, in comparison to the amniotic fluid index values of the normal curve in percentiles 2.5 and 10 for different gestational ages.

Results: the maximum pool depth had a poor sensitivity to diagnose oligohydramnios when 10 and $20 \mathrm{~mm}$ were used as cut-offs, and good sensitivity and specificity when $30 \mathrm{~mm}$ was used, in comparison to the amniotic fluid values in percentiles 2.5 and 10. The best sensitivity and specificity of the maximum pool depth were when found using a $30 \mathrm{~mm}$ cut-off in comparison to 2.5 percentile to diagnose oligohydramnios.

Conclusions: the capacity to diagnose oligohydramnios by the measure of the maximum pool depth is satisfactory only with the cut-off of $30 \mathrm{~mm}$.

KEY WORDS: Oligohydramnios. Amniotic fluid index (AFI). Pregnancy.

\section{Referências}

1. Resende CMC. Artefatos em ultra-sonografia e suas bases fisicas. Rio de Janeiro: Revinter; 1987.

2. Brace RA, Wolf EJ. Normal amniotic fluid volume changes throughout pregnancy. Am J Obstet Gynecol 1989; 161:382-8.

3. Hill LM. Abnormalities of amniotic fluid. In: Nyberg DA, Mahony BS, Pretorius DH, editores. Diagnostic Ultrasound of Fetal Anomalies: Text and Atlas. St. Louis: Mosby-Year Book; 1990. p. 38-66.

4. Wagner G, Fuchs F. The volume of amniotic fluid in the first half of pregnancy. J Obstet Gynaecol Br Commonw 1962; 69:131-6.

5. Gadd RL. The volume of the liquor amnii in normal and abnormal pregnancies. J Obstet Gynaecol Br Commonw 1966; 73:11-22.

6. Beischer NA, Brown JB, Townsend L. Studies in prolonged pregnancy. Am J Obstet Gynecol 1969; 103: 496-503.

7. Moore TR, Cayle JE. The amniotic fluid index in normal human pregnancy. Am J Obstet Gynecol 1990; 162:1168-74.

8. Manning FA, Platt, LD, Sipos, L. Antepartum fetal evaluation: development of a fetal biophysical profile. Am J Obstet Gynecol 1980; 136: 787-95.

9. Phelan JP, Smith CV, Broussard P, Small M. Amniotic fluid volume assessment with the four-quadrant technique at 36-42 weeks gestation. J Reprod Med 1987; 32:540-2.

10. Croom CS, Banias BB, Ramos-Santos E, Devoe LD, Bezhadian A, Hiett AK. Do semiquantitative amniotic fluid indexes reflect actual volume? Am J Obstet. Gynecol 1992; 167:995-9. 
11.Bottoms SF, Welch RA, Zador IE, Sokol RJ. Limitations of using maximum vertical pocket and other sonographic evaluations of amniotic fluid volume to predict fetal growth: Technical or physiologic? Am J Obstet Gynecol 1986; 155:154-8.

12.Moore TR. Superiority of the four-quadrant sum over the single-deepest-pocket technique in ultrasonographic identification of abnormal amniotic fluid volumes. Am J Obstet Gynecol 1990; 163:762-7.

13.Manning FA, Morrison I, Lange IR, Harman CR, Chamberlain PF. Fetal assessment based on fetal biophysical profile scoring: experience in 12,620 referred high risk pregnancies. I. Perinatal mortality by frequency and etiology. Am J Ostet Gynecol 1985; 151:343-50.

14.Font G, Solari M. Perfil biofísico fetal. In: Pastore AR, Cerri GG, editores. Ultra-Sonografia Obstetrícia. Ginecologia. São Paulo: Sarvier; 1997. p.195-9.
15.Manning FA, Baskett T, Morrison I, Lange IR. Fetal biophysical profile scoring: a prospective study in 1184 high risk patients. Am J Ostet Gynecol 1981; 140: 289-94.

16.Jeng CJ, Jou TJ, Wang KG, Yang YC, Lee YN, Lan, CC. Amniotic fluid index measurement with the four-quadrant technique during pregnancy. J Reprod Med 1990; 35:674-7.

17.Grannum PAT, Berkowitz, RL, Hobbins JC. The ultrasonic changes in the maturing placenta and their relationship to fetal pulmonic maturity. Am J Obstet Gynecol 1979; 133:915-22.

18.Perrotti MRM. Curva dos valores do índice de líquido amniótico em gestantes normais [Dissertação de Mestrado]. Campinas: Faculdade de Ciências Médicas. UNICAMP; 1998.

19.Halperin ME, Fong KW, Zalev AH, Goldsmith $\mathrm{CH}$. Reliability of amniotic fluid volume and maternal plasma volume estimation from ultrasonograms: intraobserver and interobserver variation before and after the establishment of criteria. Am J Obstet Gynecol 1985; 153: 264-7.

\section{CONGRESSO BRASILEIRO SOBRE MATERNIDADE SEGURA E SAÚDE REPRODUTIVA}

\section{Período: 14 a 18 Novembro de 2000}

\section{Local: Brasília}

\section{ORGANIZAÇÃO: SGOB}

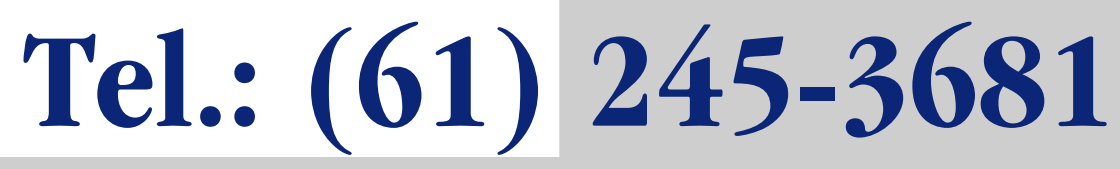

